Table 3. Fermentation of various carbohydrates, alcohols and glucosides by butyric acid bacilli

\begin{tabular}{|l|c|c|}
\hline \multirow{2}{*}{ Carbon source } & \multicolumn{2}{|c|}{ Number of strains forming } \\
\cline { 2 - 3 } & Acid & Gas \\
\hline Glycerol & 121 & 120 \\
Sorbitol & 179 & 187 \\
Mannitol & 235 & 230 \\
Xylose & 212 & 195 \\
Arabinose & 221 & 186 \\
Rhamnose & 97 & 58 \\
Fructose & 260 & 260 \\
Glucose & 260 & 260 \\
Mannose & 259 & 259 \\
Galactose & 241 & 228 \\
Sucrose & 224 & 215 \\
Maltose & 229 & 202 \\
Iactose & 211 & 186 \\
Raffinose & 197 & 195 \\
Inulin & 179 & 173 \\
Dextrin & 185 & 205 \\
Starch & 166 & 172 \\
Salicin & 197 & 163 \\
& & \\
\hline
\end{tabular}

the same type and the same extent as that found for the other properties examined. For example, one and the same strain on repeated examination of one and the same carbon source may show no attack, attack with production of acid only, attack with evolution of gas only, and finally, attack with formation of both acid and gas.

We therefore agree with McCoy et al. ${ }^{4}$, that it is impossible to use the fermenting power for the classification of butyric acid bacteria not liquefying gelatin.

According to McCoy et al. ${ }^{4}$ the butyric acid bacilli not liquefying gelatin may be divided into two groups, called Bacillus saccharobutyricus-type and Clostridium pasteurianum-type, respectively. Strains belonging to the former group liquefy the starch gel of corn-broth, whereas those of the latter group do not. The results obtained in examining our 260 freshly isolated strains by means of the method described by the authors mentioned may be sum. marized as follows. Between absolutely inactive strains on one side, and strongly active strains on the other side, there are intermediate types attacking the gel only partially. No obligate parallelism could be demonstrated between starch dissimilation in cornbroth and the breakdown of this polysaccharide in peptone-meat-broth. Furthermore, the power of attacking starch showed the same variability as that observed in the other properties of the strains examined. Thus our results do not support a classification of the butyric acid bacteria not liquefying gelatin on the basis of the changes brought about in corn-broth.

On examination for nitrogen-fixing power in Winogradsky's solution, our pure cultures were found to vary considerably, qualitatively as well as quantitatively. A large number of strains which in their properties, for example, growth on gelatin medium, fermentation of carbohydrates, higher alcohols, etc., are not identical with Winogradsky's organism, induce a turbulent fermentation in the solution without nitrogen compounds.

From the results discussed above the conclusion may be drawn that it is impossible to base a reasonable classification of the butyric acid bacilli not liquefying gelatin on differences in the properties dealt with in the present investigation.

${ }^{1}$ Winogradsky, S., Cbl. Bakt., II. Abt., 9, 43 (1902).

' Svartz, Nanna, J. Inf. Dis., 47, 138 (1930).

san Beynum, J., and Pette, J. W., Cbl. Bakt., II. Abt., 93, 198 (1935-36).

- McCoy, Elizabeth, Fred, E. B., Peterson, W. H., and Hastings, E. G. J. Inf. Dis., 46, 118 (1930).

\section{RADIO COMMUNICATION AND THE IONOSPHERE}

$\mathrm{T}$ HE great strides made in the development of long-distance radio communication and broadcasting in the past ten years or so have involved the combined efforts of a large number of scientific workers, engineers and operators. These and older students of the subject have become aware of the fact that the transmission of radio waves to distances beyond the horizon and even right round the earth is only possible because of the existence of the ionosphere, which is that portion of the earth's atmosphere which becomes electrically conducting by ionization chiefly under the influence of ultra-violet radiation from the sun. But while this conducting region reflects radio waves and so returns them to the earth at a distant point, it does not behave as a simple metallic reflecting sheet in the same position at all times and seasons. It is rather in the nature of a series of partially conducting layers, one above the other, each varying in height and conductivity with time, and the lower ones sometimes shielding the upper ones from radio waves transmitted upwards from the earth's surface.

On account of the somewhat complicated nature of this subject, it was found necessary during the War to provide, in a suitable booklet, a background knowledge of the fundamental principles of the transmission of radio waves through the ionosphere for those interested in short-wave communication. Such a book was written by Mr. G. Millington of the Marconi Wireless Telegraph Company on behalf of the Inter-service Ionosphere Bureau and at the suggestion of the Admiralty. It was extensively used by Service Departments during the War, and it seemed to the Admiralty and the Department of Scientific and Industrial Research that the information it contained should be made more widely available for radio technical workers and students. It has therefore now been reprinted with only slight modification as "Radio Research Special Report No. 17-Fundamental Principles of Ionospheric Transmission" (published for the Department of Scientific and Industrial Research by H.M. Stationery Office, 1s. $6 d$. (U.S.A., 55 cents), by post $1 s .8 d$.).

This report describes the main properties of the ionosphere as a medium in which radio waves from a transmitting station are bent or reflected back to a distant receiving station. The effect of the angle at which the waves are projected from the earth toward the ionosphere is described, together with the influence of sunlight on the transmission, with its resulting possibilities for communication. The text is of a descriptive theoretical nature, and the use of mathematics has been kept to a minimum so that the treatment can be followed by as wide a circle as possible of those interested in this subject.

The book is primarily meant for those who can supplement the reading of it by attending a training course, and perhaps even more for those whose task it is in these days to conduct such courses, and who until now have been handicapped by the lack of any book of the scope of the present one. No reference is made to actual experimental results, such as the values of the heights and critical frequencies of the ionospheric layers, and the diurnal and seasonal variations of these values. The publication admirably fulfils, however, its intended purpose of providing a basis for appreciating and understanding the practical results that can be found elsewhere. 